\title{
BEHAVIOUR ANALYSIS AND SOCIAL SKILLS TRAINING: SOME POINTS TO CONSIDER
}

\author{
ALAN RALPH \\ Murdoch University
}

ABSTRACT

Growing interest in social skills training has resulted in the development of a wide range of programmes. Concurrently, a wealth of conceptual and empirical studies has appeared in the literature. This paper presents a model of social skills training, based on recent research, which can be applied in a wide variety of settings. Initial assessment, training format and outcome evaluation are all discussed. In addition, two alternative training strategies are considered which contrast cognitive and behavioural variables as factors for increasing the probability of generalization. Finally, the issue of maintenance is also considered.

\section{SOCIAL SKILLS TRAINING}

Social skills training refers both to a specific set of procedures which typically include modelling, verbal instructions, behaviour rehearsal (role play), feedback and reinforcement (Sarason \& Sarason, 1981; Van Hasselt, Kazdin, Hersen, Simon \& Mastantuono, 1985) and at the same time to a widening range of programmes aimed at any behaviour which might conceivably be included under this heading. Such a wide scope decreases the usefulness of the term and other terms are now needed to describe particular areas of concern (Alexander, 1982; McFall, 1982).

This discussion will focus on interpersonal communication skills within a context of educational settings although the model has a much wider applications.

\section{BEHAVIOUR ASSESSMENT}

Usually, the desire to implement a special training programme arises from the perception of a teacher, parent or other responsible adult that a problem exists which is not responsive to less intrusive, behaviour management techniques. Often the problem is also a general one in that it is observed to occur across time, situations and people. This typically results in negative labels being applied such as aggressive and disruptive or, isolated and withdrawn. However, these global terms do not indicate what specific behaviour contributes to these judgements about competency in interpersonal interactions.

There has been much debate in the literature concerning the identification of these behavioural components. Argyle, Graham, Campbell \& White (1979) and Graham, Argyle, Clarke \& Maxwell (1981) investigated common rules and behavioural elements which apply across a number of different situations, while Conger and Farrell (1981) explored the behavioural referents of conversational skill and anxiety. Jones, Hobbs and Hockenbury (1982) identified specific social skill deficits associated with loneliness and Royce (1982) suggested a number of skills which identified persons adjudged to be competent in heterosocial interactions although there were differences between males and females. Dow (1985) also identified a number of behaviours considered to be important in heterosocial interactions, although some were different to those identified by Royce (1982) and Jones et al (1982). Ralph (Note 1) demonstrated that some behaviour, especially verbal behaviour, is not stable across time and setting. Therefore, although a number of reliable observation methods have been developed, efforts to specify the micro-or molecularlevel behavioural components which could be used to construct training programmes for general use have largely failed.

An alternative strategy has recently been proposed which incorporates the reliability of the micro-level approach to assessment and the more meaningful social validity of the global-level approach. Sometimes termed 'midi'-level measurement (Boice \& Monti, 1982; Monti, Kolko, Fingeret \& Kwick, 1984; Monti, Boice, Fingeret, Zwick, Kolko, Munroe \& Grunberger, 1984), this strategy has a number of features particularly wellsuited for use in the assessment of social competency. Firstly, regular observers (e.g., class teachers) and competent participants (e.g., peers) are asked for their personal observations of the problems associated with the individual identified as lacking in competence. A number of descriptive items would thus be obtained which might consist of a list similar to the following:

'Isolated child'. Never asks to join in; Doesn't play with others; Hovers around the edges a lot; Doesn't answer questions; Runs off when others approach; Always alone.

'Aggressive child'. Doesn't take notice of what others say; Pushes in; Takes over; Shouts at others if they disagree; Gets angry if he doesn't win; Hits others during conflicts.

A number of items is then selected to construct the behaviour observation measure (Birnbrauer, Note 2). For instance the item 'doesn't play with others' might be used to construct the following scale:

Score Behaviour observed

3 Played with more than 1 other child for more than $50 \%$ of the time.

2 Played with more than 1 other child, but for less than $50 \%$ of the time.

OR Played with only one child for more than $50 \%$ of the time.

$1 \quad$ Played with only one child, but for less than $50 \%$ of the time.

$0 \quad$ Never played with any other child at all. 
Although this strategy has not featured strongly in the literature as yet, it is similar to validation procedures proposed by DiLorenzo and Foster (1984), Holmes, Hansen and St. Lawrence (1984), and Kolko and Milan (1985).

Details for the different levels of the scale are obtained by observing various competent individuals to identify how they behave in the settings that are problematical for the incompetent child, e.g., joining in games during recess, remaining friendly during competitive activities, or sharing during group project sessions. If observation is difficult or impractical, details of the behaviour may be elicited by careful questioning about typical responses in the situations of interest, or by simulating them in a role-play type format (Bates, 1980; Kolko \& Milan, 1985; Minkin, Braukmann, Minkin, Timbers, Timbers, Phillips \& Wolf, 1976).

In the case of the example presented above, interest would be focussed on the amount of time competent individuals spend playing with other children in different circumstances. On its own, this may not be a particularly useful measure of social competency, but in conjunction with a number of other measures similarly constructed, it has the potential to provide a comprehensive, relevant and easy-to-use behavioural observation measure.

If competent performance on a given item is not as clearcut as in the example given above, then it will be necessary to rank order the range of typical responses obtained in each situation from most to least competent so as to provide a graded measure which is sensitive to improvements that fall short of complete mastery (Bates, 1980). For instance, the following eight 'rules' of 'asking to join in a game' might be elicited by watching others or by asking participants to state them.

Wait for an appropriate moment before asking.

Ask someone you know.

Get his or her attention before you ask.

Look at his or her face before you speak to them.

Verbally express interest and ask directly if you can join in.

If refused, ask again.

If you don't know how to play, ask someone.

Don't barge straight in, play around on the edges until you get the idea.

However, some of these rules might be more important than others, while some might be safely omitted provided that others were included. In order that the scale might be constructed as accurately as possible, it is best to have several different people judge the items independently. In this way a socially valid scale can be constructed for each item. The finished scale for the above example might be as follows:

\section{Score Behaviour observed}

3 Gets someone's attention and asks appropriately for permission to join the game.

2 Gets someone's attention, asks for permission to join in, but does so inappropriately.
1 Joins in without asking, but doesn't disrupt the game very much.

OR Approaches someone, but fails to get their attention or ask to join in.

0 Barges in without asking and disrupts the game.

OR Doesn't approach anyone at all.

Once completed, the result is a validated, custommade behaviour observation measure consisting of a number of relevant items, each comprising a series of graded behavioural descriptions, which are observable in a variety of settings.

The degree of reliability of the measure will depend upon how it is used and this can only be established by having two or more observers rate the same child over the same time interval. Although the use of such measures is not yet widely reported in the literature, it is recommended that they should be used by people who are in close proximity to the person being observed and following a previously specified period of time e.g., after recess, or other periods when the behaviour has previously been of concern. Another advantage of such a measure is that it may be used by relatively unskilled observers on a regular basis without the need for intensive observation periods or complex scoring procedures. Total scores, obtained during repeated uses of the scale, represent an index of overall behaviour change, and individual scores on the separate items are used to identify specific changes.

\section{TRAINING FORMAT}

There are four main decisions which must be made before training can begin. The first concerns the goals of the programme as characterised by the establishment of specific behavioural criteria for competency. This is provided for in the construction of the rating scales (Kazdin, 1985). The second concerns the methodology for the practical implementation of the programme. The most effective training procedure consists of a multi-faceted approach which includes modelling, verbal instruction, behaviour rehearsal, feedback and reinforcement (Van Hasselt et al, 1985). The third decision concerns the content of the programme. The basis for this is established by virtue of the observations made during the construction of the behaviour assessment measure. However, a more detailed analysis of the behaviour required must be carried out in order to discover the variations that exist in the real world that a competent child would be able to cope with (Ralph \& Birnbrauer, 1985). The fourth and final decision concerns the therapeutic emphasis of the training approach. It is here that the greatest area of debate still exists and two similar approaches with different emphases have been selected for use within this model.

In general, the use of the multi-faceted procedure referred to above has been consistently associated with improvements in social competency in the training setting, which have subsequently not occurred consistently outside this setting (Bates, 1980; Roessler \& Lewis, 1984).

One procedure which has been shown to increase the likelihood for such improvements involves making the training situation as much like the outside world as 
possible (Stokes \& Baer, 1977). This requires relevant knowledge of the outside world. Some of this is acquired during the construction of the behaviour observation measure. Once this information has been collected, a variety of scenarios is scripted with a series of verbal exchanges (Kolko \& Milan, 1985; Ollendick, Hart \& Francis, 1985) to represent typical problematical incidents. These scenarios are then used as contexts for training purposes. Although the construction of these scenarios is initially rather time-consuming, once several have been devised they may be readily adapted for new situations as needed.

Unfortunately it is not possible to create a training environment which is exactly like the real world. People, places, words, incidents and outcomes will all vary in diverse and unpredictable ways and the individual must be able to cope with these differences. Both of the training approaches selected have proposed different procedures in an attempt to solve this problem.

The first approach is a modification of the basic skills training approach (Bates, 1980; Kelly, Wildman, Urey \& Thurman, 1980) and is described as a 'general-case training' approach (e.g., Horner \& McDonald, 1982; Horner, Sprague \& Wilcox, 1982). The second approach is best described as a more cognitively oriented 'problem solving' approach (e.g., Thacker, 1982; Wallace, 1982). In respect of the problem of 'generalization' referred to above, the general-case training approach emphasises the performance of a wide range of observable behavioural skills, whereas the problem-solving approach emphasises the acquisition of cognitive skills. The common elements shared by each approach are illustrated below in the context of an aggressive or isolated child wishing to join an existing group activity:

\section{PHASE 1: STIMULUS PERCEPTION.}

This phase is primarily concerned with ensuring that the child perceives the stimulus information correctly e.g., does the child know the children involved, what are their names, what game are they playing, who is the leader? The possibility of errors in this phase being associated with sensory impairment should be investigated before proceeding.

\section{PHASE 2: RESPONSE SELECTION}

This phase is concerned with the selection of an appropriate response from the range of typical responses which competent children make, e.g., "Hi Tim, is it O.K. for me to join in?"

\section{PHASE 3: STYLE OF RESPONDING}

This phase is concerned primarily with making the selected response in an appropriate or competent manner, e.g., get the attention of the leader or a known friend, look at his or her face, speak clearly, wait for the reply, respond appropriately to the reply.

\section{GENERAL CASE TRAINING VS. PROBLEM SOLVING}

In the general-case training approach, the appropriate response for any given scenario is selected and modelled by the trainer (e.g., getting someone's attention and asking appropriately to join in). The participant is then instructed to copy the trainer's behaviour until he or she achieves a predetermined criterion (e.g., maximum score on that item on the observation measure on three successive occasions). Then different examples requiring alternative responses are presented and practised in the same way across a number of similar scenarios. For instance, repeating the question if the person asked doesn't reply, asking again if the first request is refused, asking someone else if the first person asked is unhelpful and using different types of games e.g., football, chasey, hide and seek, marbles and leapfrog depending on the age and typical activity range of the peer group in question.

In this way the participant gains practice, feedback and reinforcement for different appropriate ways of dealing with different examples of a given problem, e.g., joining in an existing group activity. This also includes learning to identify responses which are not appropriate.

In the problem solving approach, the participant is asked to generate his or her own responses to the same situation. The aggressive child's first suggested response might be to 'just barge right in and take over'. If the child fails to suggest one, the list of previously generated alternative responses is presented and he or she is asked to select one. Children are also asked to identify their short- and long-term goals in the interaction and to predict the likely outcome associated with the response they have chosen. In this instance, the short-term goal might be expressed as 'getting to join in the game' and the long-term goal might be expressed as 'making more friends'. The aggressive child might suggest that 'barging in' will help to achieve both these goals. He or she can then practise making the chosen response and subsequently receive feedback concerning whether the specified goals were achieved and whether the actual outcome matched the initial predictions. In this case, the training scenario which they role play might show that one or neither of the goals is achieved by adopting the 'barge in and take over' strategy. This process is then repeated using a number of different responses (e.g., asking, demanding, bribing, threatening), but with each one, the emphasis is on rehearsing this problem-solving routine and predicting and getting feedback on the actual outcome as experienced in the role-play scenarios.

Thus in the former approach more time is spent practising different ways of dealing appropriately with a range of different events, whereas in the latter approach more time is spent rehearsing cognitive strategies which can be put into effect when faced with a range of different events. These are the contrasting strategies adopted by each approach which are believed to increase the probability of the appropriate behaviour occurring outside the training setting.

Throughout all phases, feedback may be received either verbally or from replays of video-tape or audiotape recordings. Reinforcement usually consists of praise plus tangible reinforcers depending on the age and interests of the participants. The programme may consist of a varied number of essentially similar sessions with scenarios being repeated until a predetermined criterion is attained, or until the time allocated for each participant or scenario is exhausted during any given session. 
Bramston and Spence (1985), working with intellectually handicapped adults, compared a basic skills-training approach with a problem-solving approach and found that each was associated with improvements of the respective target behaviours relative to two control groups, but that neither brought about substantial improvements in other settings and maintenance was poor. However, the assessment measures were not constructed in accordance with the precepts outlined in this paper. In addition, the range of examples presented was limited, unlike the general case approach, and the content of the training scenarios was not described.

Sarason and Sarason (1981) used a combined skills training and problem solving approach to improve behaviour with high school students and obtained some encouraging results on measures which included an apparently unrelated job interview four months later and school data on absence from school, tardiness and reports for behaviour problems during the following year. Again, a wide range of different examples was not programmed, nor is it possible to separate the relative contributions of the skills training and problem-solving components.

Any attempt to combine the two approaches in order to get the 'best of both worlds', will require a longer training time. It is therefore important that the respective merits of each be fully evaluated in order to select the most effective treatment procedure. Ollendick (Note 3 ) has research data which suggest that isolated and withdrawn children may respond better to the problemsolving approach, whereas aggressive or disruptive children may respond better to a skills training approach. More research of this type will be needed before a clear picture merges. There is also a scarcity of research reporting the effects of general-case training in interpersonal interactions which must be addressed.

\section{EVALUATION}

The final stage of the model is concerned with evaluating behaviour in the real world. Many attempts have been made to construct simulated tests in vivo (Kelly, Wildman \& Berler, 1980), tests involving deception (Montgomery \& Haemmerlie, 1982) or specially developed role plays (Ollendick, Hart \& Francis, 1985). Many of these have failed to demonstrate predictive value regarding behaviour in the real world (Kazdin, Matson \& Esveldt-Dawson, 1983; McNamara \& Blumer, 1982). Once again, the problem is one of accurately replicating, in the training setting, the critical characteristics associated with problem situations in the real world. Participation in a simulated or role-played test may be useful during training to determine whether the skill can actually be performed competently or not, but it is of little value in predicting whether it will be performed competently in another setting at another time. The only really satisfactory solution is the use of direct observation in the setting(s) where the behaviour is required, as previously described. The availability of a reliable and valid measure, such as the one described above, is therefore indispensable in determining whether significant and substantial improvement has taken place (Kazdin, 1985; Kupke, Calhoun \& Hobbs, 1979).

\section{MAINTENANCE}

Maintenance is concerned with whether a newly acquired skill continues to be produced outside the training setting once it has occurred there. The behaviour principle which states that behaviour is the result of its consequences is of prime importance here. If this newly acquired behaviour is not sufficiently reinforced when it occurs, then one may expect that it will be extinguished quite quickly. Should extinction occur, it may be because the behaviour is functionally irrelevant in this situation. This usually results from a poor initial assessment and it is to avoid this very outcome that a prior analysis of typical competent behaviour in the real setting is carried out.

Alternatively, it may be that those in control of the reinforcers have become so used to having the child behave in a certain way that they do not perceive the changes and consequently fail to reinforce them. It is in just such a situation that the training programme should continue to support the behaviour during this transition period. If the analysis has been carried out correctly and the behaviour can be reinforced at a distance by the trainer (Ballard \& Jenner, 1981; Ralph \& Birnbrauer, in press), it will eventually be noticed in the real world, perhaps assisted by some subtle prompting, and subsequently reinforced naturally and consequently maintained.

Occasionally a situation may be encountered where support cannot be maintained during the transition period, or where this period is unduly extended due to the refractory behaviour of key people in the child's environment. It may then be in that child's best interest to move to a new environment where such powerful stigma do not act to extinguish newly acquired behaviour.

\section{SUMMARY}

A model for assessing, training and evaluating competency in interpersonal interactions has been discussed. The critical points consist of (1) observation of and consultation with persons in or familiar with the identified problem settings so as to obtain meaningful descriptions of the problem behaviour; (2) the generation and ranking of a range of exemplars which typify competent and incompetent responding in these settings; ( 3 ) the use of this information to construct a reliable and socially valid behaviour observation measure; (4) the scripting of a number of scenarios which provide relevant contexts for use in training; (5) the use of a modelling, verbal instruction, behaviour rehearsal, feedback and reinforcement training format; (6) the choice of a general-case or a problem-solving emphasis (or a combination of both) to increase the probability of the newly acquired behaviour occurring outside the training setting; (7) the continued use of the behaviour observation measure outside the training setting to test the efficacy of the training; and (8) continued feedback and delayed reinforcement during the transition stage to increase the prospects for maintenance of newly acquired behaviour.

\section{REFERENCE NOTES}

1. Ralph, A. The reliability and social validity of measures of social skills. Paper presented at the Ninth National Conference of the Australian Behaviour Modi- 
fication Association, Sydney, May 1986.

2. Birnbrauer, J.S. Personal communication.

3. Ollendick, T.H. The identification and treatment of childhood and adolescent disorders. Paper presented at a meeting of the Australian Behaviour Modification Association, Perth, June, 1986.

\section{REFERENCES}

Alexander, R. (1982). The identification of social skills: An analytical review of current assessment practices in social skills training. Occasional Papers of the Division of Educational and Child Psychology of the British Psychological Society, 6, 14-21.

Argyle, M., Graham, J.A., Campbell, A. \& Whlte, P. (1979). The rules of different situations. New Zealand Psychologist, 8, 13-22.

Ballard, K.D. \& Jenner, L. (1981). Establishing correspondence between saying and doing as a procedure for increasing the social behaviours of two elementary school children. The Exceptional Child, 28, 55-63.

Bates, P. (1980). The effectiveness of interpersonal skills training on the social skill acquisition of moderately and mildly retarded adults. Journal of Applied Behaviour Analysis, 13, 237-248.

Boice, R. \& Monti, P.M. (1982). Specification of nonverbal behaviours for clinical assessment. Journal of Nonverbal Behaviour, 7, 79-94.

Bramston, P. \& Spence, S.H. (1985). Behavioural versus cognitive social-skills training with intellectually handicapped adults. Behaviour Research and Therapy, 23, 239-246.

Conger, J.C. \& Farrell, A.D. (1981). Behavioural components of heterosocial skills. Behaviour Therapy, 12, 41-55.

DiLorenzo, T.M. \& Foster, S.L. (1984). A functional assessment of children's ratings of interaction patterns. Behavioural Assessment, 6, 291-302.

Dow, M.G. (1985). Peer validation and idiographic analysis of social skill deficits. Behaviour Therapy, 16, 76-86.

Graham, J.A., Argyle, M., Clarke, D. \& Maxwell, G. (1981). The salience, equivalence, and sequential structure of behavioural elements in different social situations. Semiotica, 35, 1-27.

Holmes, M.R., Hansen, D.J. \& St. Lawrence, J.S. (1984). Conversational skills training with aftercare patients in the community: Social validation and generalization. Behaviour Therapy, 15, 84-100.

Holmes, R.H. \& McDonald, R.S. (1982). Comparison of single instance and general case instruction in teaching a generalized vocational skill. Journal of the Association for the Severely Handicapped, 8, 7-20.

Horner, R.H., Sprague, J. \& Wilcox, B. (1982). General case programming for community activities. In $\mathrm{B}$. Wilcox \& I. Bellamy (Eds.), Design of high school programmes for severely handicapped students. Baltimore: Paul H. Brookes.

Jones, W.H., Hobbs, S.A. \& Hockenbury, D. (1982). Loneliness and social skill deficits.Journal of
Personality and Social Psychology, 42, 682-689.

Kazdin, A.E. (1985). Selection of target behaviours: The relationship of the treatment focus to clinical dysfunction. Behavioural Assessment, 7, 33-47.

Kazdin, A.E., Matson, J.L. \& Esveldt-Dawson, K. (1984). The relationship of role-play assessment of children's social skills to multiple measures of social competence. Behaviour Research and Therapy, 22, 129-139.

Kelly, J.A., Wildman, B.G. \& Berler, E.S. (1980). Small group behavioural training to improve the job interview skills repertoire of mildly retarded adolescents. Journal of Applied Behviour Analysis, 13, 461-471.

Kelly, J.A., Wildman, B.G., Urey, J.R. \& Thurman, C. (1980). Group skills training to increase the conversational repertoire of retarded adolescents. Child Behaviour Therapy, 1, 323-336. 